\title{
Photogalvanic effects in quantum wells
}

\author{
S.D. Ganichev $v^{\mathrm{a}, \mathrm{b}, *}$, E.L. Ivchenko ${ }^{\mathrm{b}}$, W. Prettl $^{\mathrm{a}}$ \\ ${ }^{a}$ Institut fur Exp. und Angew. Physik, Universität Regensburg, D-93040 Regensburg, Germany \\ b A.F. Ioffe Physico-Technical Institute, 194021 St. Petersburg, Russia
}

\begin{abstract}
Circular and linear photogalvanic effects induced by far-infrared radiation have been investigated in both n-type and p-type quantum wells (QWs) of various point symmetry groups. The circular photogalvanic effect arises due to optical spin orientation of free carriers in QWs which results in a directed motion of free carriers in the plane of a QW perpendicular to the direction of light propagation. Due to selection rules the direction of the current is determined by the helicity of the light and can be reversed by switching the helicity from right to left. (C) 2002 Elsevier Science B.V. All rights reserved.
\end{abstract}

Keywords: Photogalvanic effect; Spin orientation; Quantum wells

\section{Introduction}

The spin of electrons and holes in condensed-matter physics is an intensively studied quantum mechanical property as it is the decisive ingredient for spintronic devices $[1,2]$. Optical orientation by applying circularly polarized light is frequently used to prepare an ensemble of spin polarized carriers [3]. The experiments described below show that in low-dimensional systems with band splitting in $\boldsymbol{k}$-space due to $\boldsymbol{k}$-linear terms in the Hamiltonian optical excitation leads not only to a spin polarized ensemble of electrons but also to a photogalvanic current whose direction depends on the predominant spin orientation. The observed photocurrent flows in the quantum well (QW) perpendicular to the direction of the incident circularly

\footnotetext{
* Corresponding author. Institut fur Exp. und Angew. Physik, Universitat Regensburg, D-93040 Regensburg, Germany. Tel.: +49-941-943-2050; fax: +49-941-943-2177.

E-mail address: sergey.ganichev@physik.uni-regensburg.de (S.D. Ganichev).
}

polarized light. The effect is quite general and has been observed for all semiconductor systems investigated.

\section{Experimental technique and results}

The experiments were carried out on heterostructures belonging to two different classes of symmetry. Higher symmetric structures were $\left(\begin{array}{llll}0 & 0 & 1\end{array}\right)$-grown n-InAs QWs of $15 \mathrm{~nm}$ width and (001)-grown n-GaAs/AlGaAs single heterojunctions. These structures can belong to two point groups, either $\mathrm{D}_{2 \mathrm{~d}}$ or $\mathrm{C}_{2 \mathrm{v}}$. Our measurements showed that all samples investigated here belong to the group $\mathrm{C}_{2 \mathrm{v}}$. Structures of the lower symmetry $\mathrm{C}_{\mathrm{s}}$ were (113)A MBE grown $\mathrm{p}-\mathrm{GaAs} / \mathrm{AlGaAs}$ single $\mathrm{QWs}$ and multiple QWs (MQW) containing 20 wells of $15 \mathrm{~nm}$ width. Samples with free-carrier densities of about $10^{11}$ $\mathrm{cm}^{-2}$ were studied in the range from liquid helium to room temperature. Two pairs of ohmic contacts were centered along opposite sample edges. For optical excitation we used a high-power far-infrared 


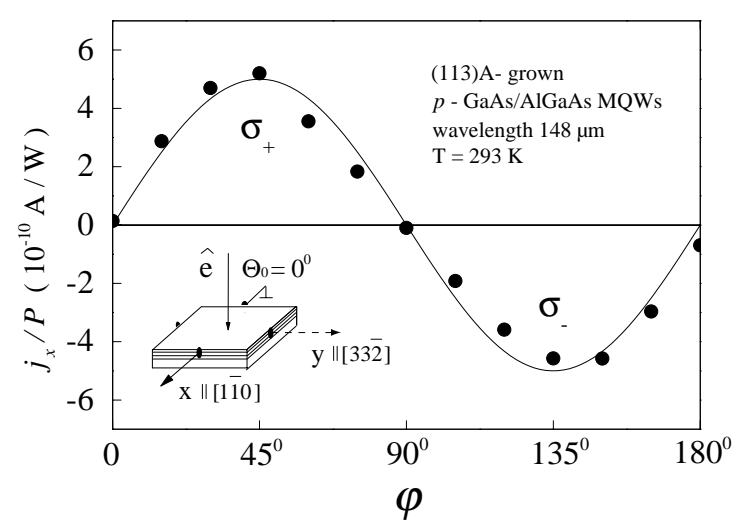

Fig. 1. Photocurrent $j_{x}$ in (1 113 )-grown GaAs QWs of $\mathrm{C}_{\mathrm{s}}$ symmetry normalized by the light power $P$ as a function of the phase angle $\varphi$ defining helicity.

pulsed $\mathrm{NH}_{3}$ laser optically pumped by a TEA $\mathrm{CO}_{2}$ laser which yields strong linearly polarized emission at wavelengths between 35 and $280 \mu \mathrm{m}$ [4]. The radiation induces indirect optical transitions in the lowest conduction subband of our n-type samples and direct optical transitions between heavy hole and light hole subbands in the p-type samples. The laser light polarization was modified from linear to circular using quartz $\lambda / 4$ plates. The helicity $P_{\text {circ }}$ of the incident light was varied from -1 (left-handed circular, $\left.\sigma_{-}\right)$to +1 (right-handed circular, $\sigma_{+}$) according to $P_{\text {circ }}=\sin 2 \varphi$ were $\varphi$ is the angle between the initial plane of polarization and the optical axis of the $\lambda / 4$ plate.

In samples of the lower symmetry class $\mathrm{C}_{\mathrm{s}}$ grown on a (1 113$)$-GaAs surface, the spin photocurrent can be observed under normal incidence. The reversal of the current direction when the polarization switches from left-handed to right-handed circular is shown in Fig. 1 where the current is plotted as a function of the phase angle $\varphi$. For this symmetry the direction of current is determined by the symmetry of the crystal. The current flows always along the [1 $1 \overline{1} 0]$-direction perpendicular to the plane of mirror reflection of the point group $\mathrm{C}_{\mathrm{s}}$, independent of the plane of incidence of the laser beam. In $(001)$-oriented samples of the higher symmetry class $\mathrm{C}_{2 \mathrm{v}}$ a signal proportional to the helicity $P_{\text {circ }}$ is only observed under oblique incidence and the photocurrent is perpendicular to the wave vector of the incident light (see Fig. 2).

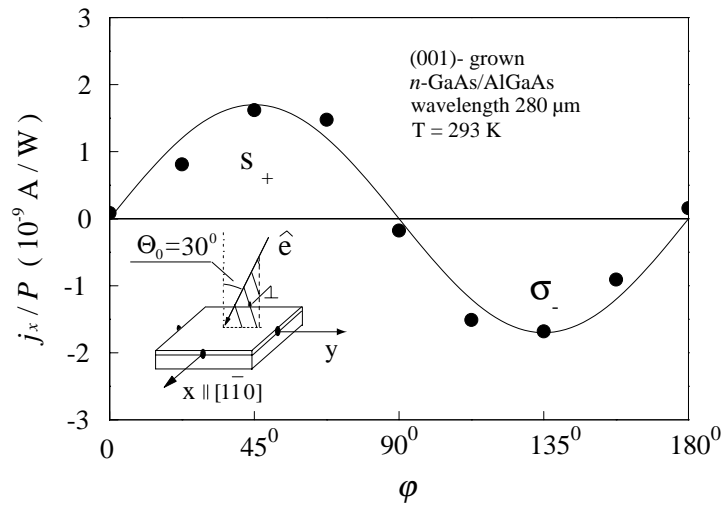

Fig. 2. Photocurrent $j_{x}$ in (0 0101$)$-grown GaAs/AlGaAs QWs of $\mathrm{C}_{2 \mathrm{v}}$ symmetry normalized by the light power $P$ as a function of the phase angle $\varphi$ defining helicity.

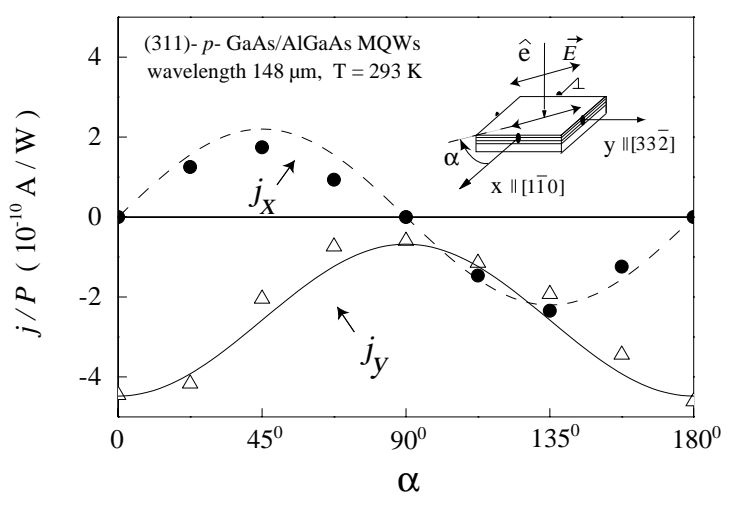

Fig. 3. Photocurrent $j$ normalized by the light power $P$ as a function of the angle $\alpha$ between the plane of linear polarization and the axis $x$. Data are obtained under normal incidence.

In addition to the helicity-dependent photocurrent a photocurrent in response to a linearly polarized radiation has been observed in both $x$ and $y$ directions. Fig. 3 presents the measured dependence of $j_{x}(\alpha)$ and $j_{y}(\alpha)$, as a function of the angle $\alpha$ between the plane of linear polarization and the axis $x$. Note that in this setup $\alpha=0$ is equivalent to $\varphi=n 90^{\circ}$ ( $n$ is integer $0, \pm 1 \ldots)$ in the setup with the $\lambda / 4$ plate. The solid and the dashed curves in Fig. 3 show the fit after $j_{x}(\alpha) \propto \sin (2 \alpha)$ and $j_{y}(\alpha) \propto\left[\chi_{+}-\chi_{-} \cos 2(\alpha)\right]$, respectively. Here $\chi_{+}$and $\chi_{-}$are constants. 


\section{Phenomenological description}

Phenomenologically, the circular photogalvanic effect (CPGE) is described by

$j_{\lambda}=\gamma_{\lambda \mu} i\left(\mathbf{E} \times \mathbf{E}^{*}\right)_{\mu}$,

where $\mathbf{j}$ is the photocurrent density, $\mathbf{E}$ is the complex amplitude of the electric field of the electromagnetic wave and $i\left(\mathbf{E} \times \mathbf{E}^{*}\right)_{\mu}=\hat{e}_{\mu} P_{\text {circ }} E_{0}^{2}$ with $E_{0}, P_{\text {circ }}, \hat{\mathbf{e}}$ being the electric field amplitude $|\mathbf{E}|$, the degree of light circular polarization and the unit vector pointing in the direction of light propagation, respectively. Photocurrent (1) depends on the sign of the circular polarization and is not induced by linearly polarized excitation. Helicity-dependent photocurrents in semiconductors have been observed in bulk Te utilizing the peculiarities of the valence band structure ('camel back') at the first Brillouin zone boundary and in bulk GaAs subjected to an external magnetic field [5]. Recently, this effect has also been observed in low-dimensional structures [6,7]. The symmetry of the second-rank pseudotensor $\gamma$ coincides with that of the tensor of gyrotropy. It has nonzero components if at least some components of polar and axial vectors transform according to the identity representation of the point group of the medium. In general, in addition to photocurrent (1), two other photocurrents can be observed simultaneously, namely

$j_{\lambda}=\chi_{\lambda \mu v}\left(E_{\mu} E_{v}^{*}+E_{v} E_{\mu}^{*}\right) / 2+T_{\lambda \mu \nu \delta} E_{\mu} E_{v}^{*} q_{\delta}$,

where $\mathbf{q}$ is the light wave vector inside the medium. The first contribution is called linear photogalvanic effect (LPGE) because it is usually observed under linearly polarized optical excitation. The linear photogalvanic effect is allowed in noncentrosymmetric media of the piezoelectric classes where there exist nonzero components of a third-rank tensor satisfying the condition $\chi_{\lambda \mu \nu}=\chi_{\lambda v \mu}$. LPGE was studied in bulk crystals (see Ref. [5] and references therein) and also can be induced in QW and quantum-wire nanostructures. This effect was recently observed experimentally in QW structures [7]. The second term on the right-hand side of Eq. (2) describes the photon drag effect $[8,9]$. It appears due to the momentum transfer from photons to free carriers and can be induced in both noncentrosymmetric and centrosymmetric systems.

The following three different point groups are relevant in connection with the experiments on photogalvanics of QW structures. A zinc blende based ( $\left.\begin{array}{lll}0 & 0 & 1\end{array}\right)$-grown $\mathrm{QW}$ with equivalent normal and inverted interfaces has $D_{2 d}$ point group symmetry. The symmetry of a $\left(\begin{array}{lll}0 & 0 & 1\end{array}\right)$-grown $\mathrm{QW}$ with unequivalent interfaces reduces to $\mathrm{C}_{2 \mathrm{v}}$ and includes two fold rotation axis $C_{2} \|\left[\begin{array}{lll}0 & 0 & 1\end{array}\right]$ and the mirror planes $\sigma_{(110)}, \sigma_{(1 \overline{1} 0)}$. In the low-symmetry QWs grown along $z \|[h h l]$ with $[h h l] \neq\left[\begin{array}{lll}0 & 0 & 1\end{array}\right]$ or [ $\left[\begin{array}{lll}1 & 1 & 1\end{array}\right]$ the point group is $\mathrm{C}_{\mathrm{s}}$ and contains only two elements, the identity and one mirror reflection plane normal to the $\left[\begin{array}{lll}1 & 1 & 0\end{array}\right]$-direction. In what follows, we use the two sets of cartesian coordinate systems. The notations $1,2,3$ or $x_{1}, x_{2}, x_{3}$ are used for the crystallographic axes [ $\left[\begin{array}{lll}1 & 0 & 0\end{array}\right],\left[\begin{array}{lll}0 & 1 & 0\end{array}\right]$ and [ [ $\left.\begin{array}{lll}0 & 0 & 1\end{array}\right]$, respectively. The coordinate axes $x, y, z$ are parallel to the directions [ 110$]$, $[l l(\overline{2 h})]$ and $[h h l]$, respectively, where $[h h l]$ is the growth axis of the QW-structure.

Since the barriers prevent confined free carriers from motion along the growth direction the index $\lambda$ in Eqs. (1) and (2) runs only over the coordinates $x$ and $y$. Then, in a system of $\mathrm{C}_{2 \mathrm{v}}$ symmetry, the CPGE is characterized by linearly independent components $\gamma_{x y}, \gamma_{y x}$ and the equation (1) reduces to

$j_{x}=\gamma_{x y} e_{y} P_{\text {circ }} E_{0}^{2}, \quad j_{y}=\gamma_{y x} e_{x} P_{\text {circ }} E_{0}^{2}$.

The same equations are also valid for the $D_{2 d}$ point group but this higher symmetry imposes the condition $\gamma_{x y}=\gamma_{y x}$ on the $\gamma$ tensor components. For both symmetries $D_{2 d}$ and $C_{2 v}$ a circular (as well as a linear) photocurrent can be induced only under oblique incidence of radiation because for normal incidence, $\hat{\mathbf{e}}=\mathbf{q} / q \|\left[\begin{array}{lll}0 & 0 & 1\end{array}\right]$ and hence $e_{x}=e_{y}=0 .{ }^{1}$ Thus rewriting the components $\gamma_{\lambda \mu}$ in the form $\gamma_{x y}=\bar{\gamma}+\tilde{\gamma}, \gamma_{y x}=\bar{\gamma}-\tilde{\gamma}$ and substituting this into Eqs. (3) we can consider the coefficient $\tilde{\gamma}$ as a signature of the symmetry reduction from $\mathrm{D}_{2 \mathrm{~d}}$ to $\mathrm{C}_{2 \mathrm{v}}$. The parameters $\bar{\gamma}, \tilde{\gamma}$ can be determined separately by measuring the photocurrent in the experimental setup where the sample can be rotated around the axis [ $\left[\begin{array}{lll}0 & 0 & 1\end{array}\right]$ and the plane of light incidence is fixed. In particular, if $\tilde{\gamma}=0$ then the circular photocurrent is perpendicular to the plane of incidence $\sigma_{0}$. In the other limiting case, $\bar{\gamma}=0$, the photocurrent direction with respect to the incidence plane changes from perpendicular to parallel as the angle between the axis $x$ and the plane of incidence $\sigma_{0}$ varies from $0^{\circ}$ to $45^{\circ}$.

\footnotetext{
${ }^{1}$ The photon drag effect in QWs is possible only under oblique incidence for any point symmetry of the system.
} 
The $\mathrm{C}_{\mathrm{s}}$ symmetry allows CPGE and LPGE for normal incidence $\hat{\mathbf{e}} \|[h h l]$ because in this case the tensors $\gamma$ and $\chi$ have the additional nonzero components $\gamma_{x z}$, $\chi_{x x y}=\chi_{x y x}, \chi_{y x x}$ and $\chi_{y y y}$. As a result, under normally incident excitation, one has for circular polarization

$j_{x}=\gamma_{x z} P_{\text {circ }} E_{0}^{2}$,

and for linear polarization

$j_{x}=\chi_{x x y}\left(E_{x} E_{y}^{*}+E_{y} E_{x}^{*}\right)$,

$j_{y}=\chi_{y x x}\left|E_{x}\right|^{2}+\chi_{y y y}\left|E_{y}\right|^{2}$.

In particular, for linearly polarized light one has $j_{x}=$ $\chi_{x x y} E_{0}^{2} \sin 2 \alpha$ and $j_{y}=\left(\chi_{+}+\chi_{-} \cos 2 \alpha\right) E_{0}^{2}$, where $\alpha$ is the angle between $x$ and the plane of polarization and $\chi_{ \pm}=\left(\chi_{y x x} \pm \chi_{y y y}\right) / 2$, which are observed in the experiment (Fig. 3).

\section{Microscopical mechanisms of CPGE}

In general, two mechanisms contribute to the CPGE, photoexcitation and scattering of photoexcited carriers [5]. The first contribution, $j^{(1)}$, is caused by the asymmetry of the momentum distribution of carriers excited in optical transitions which are sensitive to the light circular polarization due to selection rules [10]. The second contribution, $j^{(2)}$, is the result of spin relaxation of optically oriented carriers [11]. The currents $j_{1}$ and $j_{2}$ can be estimated as $j_{1,2} \simeq e w s_{0} \tau_{p} \beta \hbar$, where $e$ is the elementary charge, $w$ is the probability of photon absorption per unit time and unit volume in the case of multiple QWs (or per unit area for a single QW), $s_{0}$ is the average spin of photoexcited carriers at the moment of generation, $\tau_{p}$ is the electron momentum relaxation time, and $\beta$ is one of the coefficients $\beta_{l m}$ in the $\mathbf{k}$-linear Hamiltonian $H^{(1)}=\beta_{l m} \sigma_{l} k_{m}$ ( $\sigma_{l}$ are the Pauli matrices). Although $j_{1}$ and $j_{2}$ can be of the same order of magnitude, the physical difference between the two contributions becomes obvious after the light is switched off: $j_{1}$ decays with the carrier momentum relaxation time $\tau_{p}$ whereas the decay of $j_{2}$ is governed by the spin relaxation time $\tau_{\mathrm{s}}$ which can be much longer than $\tau_{p}$.

Microscopical consideration of the CPGE under interband optical excitation of a QW structure has been presented in Ref. [6]. Here we concentrate on the asymmetry of the momentum distribution of holes excited under direct intersubband optical transitions in p-doped $(h h l)$-grown QWs. We remind that in this case normally incident radiation of circular polarization induces a current in the $x$ direction. Let us denote the free hole states in a QW as $|v j \mathbf{k}\rangle$, where $\mathbf{k}$ is the two-dimensional wavevector, $v$ and $j$ are the hole subband and spin-branch indices. If only terms even in $\mathbf{k}$ are taken into account in the hole effective Hamiltonian, all the hole subbands $(v, \mathbf{k})$ are doubly degenerate. Allowance for terms odd in $\mathbf{k}$, linear $H^{(1)}(\mathbf{k})$ and/or cubic $H^{(3)}(\mathbf{k})$, results in the subband spin splitting so that the hole energy $E_{v j \mathbf{k}}$ becomes dependent on $j$. The photocurrent density is given by the standard expression

$j_{x}=e \sum_{v j \mathbf{k}} v_{x}(v j \mathbf{k}) f_{v j \mathbf{k}}$,

where $e$ is the elementary charge (for holes $e>0$ ), $v_{x}(v j \mathbf{k})$ is the group velocity $\hbar^{-1}\left(\partial E_{v j \mathbf{k}} / \partial k_{x}\right)$ and $f_{v j \mathbf{k}}$ is the nonequilibrium steady-state distribution function. Note that $E_{v j \mathbf{k}}$ is invariant and $v_{x}(v j \mathbf{k})$ changes its sign under the time-inversion operation transforming a spinor $\hat{\psi}$ into $K \hat{\psi} \equiv i \sigma_{y} \hat{\psi}$ ( $\sigma_{y}$ is one of the Pauli matrices) [5]. Therefore, $j_{x}$ is contributed only by the antisymmetric part of the distribution function $f_{v j \mathbf{k}}^{-}=\left(f_{v j \mathbf{k}}-f_{v \bar{j},-\mathbf{k}}\right) / 2$, where $|v \bar{j},-\mathbf{k}\rangle$ is obtained from $|v j \mathbf{k}\rangle$ by application of the operator $K$.

In the momentum relaxation time approximation we have under direct optical transitions

$$
\begin{aligned}
j_{x}= & e \sum_{v^{\prime} v j^{\prime} j \mathbf{k}} W_{v^{\prime} j^{\prime}, v j}(\mathbf{k}, \mathbf{e}) \\
& \times\left[v_{x}\left(v^{\prime} j^{\prime} \mathbf{k}\right) \tau_{p}^{\left(v^{\prime}\right)}-v_{x}(v j \mathbf{k}) \tau_{p}^{(v)}\right],
\end{aligned}
$$

where $\mathbf{e}$ is the photon polarization unit vector, $\tau_{p}^{(v)}$ is the hole momentum relaxation time in the subband $v$. The probability rate for the transition $|v j \mathbf{k}\rangle \rightarrow\left|v^{\prime} j^{\prime} \mathbf{k}\right\rangle$ is given by Fermi's golden rule

$$
\begin{aligned}
W_{v^{\prime} j^{\prime}, v j}(\mathbf{k}, \mathbf{e})= & \frac{2 \pi}{\hbar}\left|M_{v^{\prime} j^{\prime}, v j}(\mathbf{k})\right|^{2}\left(f_{v j \mathbf{k}}^{0}-f_{v^{\prime} j^{\prime} \mathbf{k}}^{0}\right) \\
& \times \delta\left(E_{v^{\prime} j^{\prime} \mathbf{k}}-E_{v j \mathbf{k}}-\hbar \omega\right),
\end{aligned}
$$

where $\hbar \omega$ is the photon energy, $M_{v^{\prime} j^{\prime}, v j}(\mathbf{k})$ is the intersubband optical matrix element proportional to the amplitude of the electromagnetic field and $f_{v j \mathbf{k}}^{0}$ is the distribution function in equilibrium. For the sake of simplicity, we assume here that the light intensity is low enough to ignore a photoinduced redistribution of the symmetrical part $f_{v j \mathbf{k}}^{+}=\left(f_{v j \mathbf{k}}+f_{v \bar{j},-\mathbf{k}}\right) / 2$. 
Physically, the CPGE can be considered as a transformation of the photon angular momenta into a translational motion of free charge carriers. In terms of Eq. (7) this means a conversion of photon helicity into a spin photocurrent which becomes possible due to spin dependent and odd-in-k terms in the free-carrier effective Hamiltonian, $H^{(1)}(\mathbf{k})+H^{(3)}(\mathbf{k})$. It is worth mentioning that the CPGE is an electron analog of a mechanical systems which transmit motion either rotatory to linear or linear to rotatory like a screw tread, a plane with a propeller or a rotating wheel on a hard surface.

\section{Terms linear and cubic in $\mathbf{k}$}

For the $(h h l)$-grown QWs the relevant terms needed to be taken into account are the terms odd in $k_{x}$ and $\hat{J}_{z}$. Allowance of them results in the energy dispersion

$E_{v j \mathbf{k}}=E_{v \mathbf{k}}^{0} \pm k_{x}\left(\beta_{v}+\alpha_{1, v} k_{x}^{2}+\alpha_{2, v} k_{y}^{2}\right)$,

where $E_{v \mathbf{k}}^{0}$ is the hole energy in the state $|v j \mathbf{k}\rangle$ when the odd terms are ignored in the hole Hamiltonian (for calculation of $E_{v \mathbf{k}}^{0}$ see, e.g. Ref. [12]). The coefficients $\alpha_{1, v}, \alpha_{2, v}, \beta_{v}$ can be related to the coefficients $c_{1}$ and $c_{3}$ of the terms in the Hamiltonians of the bulk crystal being linear and cubic in $\mathbf{k}$, respectively,

$$
\begin{aligned}
H^{(1)}= & c_{1}\left(\hat{V}_{1} k_{1}+\hat{V}_{2} k_{2}+\hat{V}_{3} k_{3}\right), \\
H^{(3)}= & c_{3}\left[\hat{J}_{1} k_{1}\left(k_{2}^{2}-k_{3}^{2}\right)+\hat{J}_{2} k_{2}\left(k_{3}^{2}-k_{1}^{2}\right)\right. \\
& \left.+\hat{J}_{3} k_{3}\left(k_{1}^{2}-k_{2}^{2}\right)\right],
\end{aligned}
$$

where $\hat{J}_{\lambda}$ are the $4 \times 4$ matrices of the angular momentum components in the basis of the states with $J=\frac{3}{2}, \hat{V}_{1}=\left[\hat{J}_{1}\left(\hat{J}_{2}^{2}-\hat{J}_{3}^{2}\right)+\left(\hat{J}_{2}^{2}-\hat{J}_{3}^{2}\right) \hat{J}_{1}\right] / 2$ etc. Transforming the coordinate system from $x_{1}, x_{2}, x_{3}$ to $x, y, z$ and applying the perturbation theory in the first-order approximation we obtain for the valence band odd-term coefficients

$$
\begin{aligned}
\beta_{v}= & \sin \theta\left(1-\frac{3}{2} \sin ^{2} \theta\right)\left(-c_{1}\left\langle v s \mathbf{k}\left|\hat{J}_{z}^{3}\right| v s \mathbf{k}\right\rangle\right. \\
& \left.+c_{3}\left\langle v s \mathbf{k}\left|\hat{J}_{z} \hat{k}_{z}^{2}\right| v s \mathbf{k}\right\rangle\right), \\
\alpha_{1, v}= & \frac{1}{2} c_{3} \sin \theta J_{z}^{(v)}, \\
\alpha_{2, v}= & -c_{3} \sin \theta\left(1+\frac{3}{2} \cos ^{2} \theta\right) J_{z}^{(v)},
\end{aligned}
$$

$J_{z}^{(v)}=\left\langle v s \mathbf{k}\left|\hat{J}_{z}\right| v s \mathbf{k}\right\rangle, \quad \hat{k}_{z}^{2}=-\frac{\partial^{2}}{\partial z^{2}}$,

where $\theta$ is the angle between the axes $[h h l]$ and $\left[\begin{array}{ll}0 & 0\end{array}\right],|v s \mathbf{k}\rangle$ is the hole state which is calculated neglecting the odd terms and symmetrical with respect to the mirror reflection in the plane $(h h l)$ as defined in Ref. [12]. The procedure used to obtain Eqs. (12) is somewhat similar to that used in Ref. [13] to obtain the terms linear in $\mathbf{k}$ from cubic terms for electrons in the conduction band of QW structures. Applying Eqs. (9), (12) one can proceed to perform numerical calculations of $j_{x}$ according to Eqs. (7) and (8).

\section{Summary}

In summary, the experiments carried out on different types of QW have shown that circularly polarized light can generate a directed electric current at room temperature. The microscopic picture given above requires that the generated current is spin polarized and suggests that the system can be considered as a source for spin-polarized currents. The effect, being sensitive to the degree of spin orientation, provides an easy access to spin dynamics in semiconductor structures $[14,15]$. In addition to the circular photogalvanic effect the linear photogalvanic effect and the photon drag effect have been observed in QWs of both nand p-type. Both photocurrents are well described by phenomenological equations derived above.

\section{Acknowledgements}

Financial support from the DFG, the RFFI, the INTAS and the NATO linkage program is gratefully acknowledged.

\section{References}

[1] S. Datta, B. Das, Appl. Phys. Lett. 56 (1990) 665.

[2] G.A. Prinz, Phys. Today 48 (1995) 58.

[3] F. Meier, B.P. Zakharchenya (Eds.), Optical orientation, Elsevier Science Publishers, Amsterdam, 1984.

[4] S.D. Ganichev, Physica B 273-274 (1999) 737.

[5] E.L. Ivchenko, G.E. Pikus, Superlattices and Other Heterostructures. Symmetry and Optical Phenomena, 2nd Edition, in: M. Cardona, P. Fulde, K.v. Klitzing, H.-J. Queisser (Eds.), 
Solid State Sciences, Springer Series, Vol. 110, Springer, Berlin, 1995, 1997 (Chapter 10).

[6] S.D. Ganichev, E.L. Ivchenko, S.N. Danilov, J. Eroms, W. Wegscheider, D. Weiss, W. Prettl, Phys. Rev. Lett. 86 (2001) 4358.

[7] S.D. Ganichev, H. Ketterl, W. Prettl, E.L. Ivchenko, L.E. Vorobjev, Appl. Phys. Lett. 77 (2000) 3146.

[8] S.M. Ryvkin, I.D. Yaroshetskii, Problemy sovremennnoi fiziki (Problems of Modern Physics), Leningrad presses de l'université Laval, Quebec, 1980.

[9] S. Luryi, Phys. Rev. Lett. 58 (1983) 2263.

[10] E.L. Ivchenko, G.E. Pikus, Sov. Phys. JETP Lett. 27 (1978) 604.

[11] E.L. Ivchenko, Yu.B. Lyanda-Geller, G.E. Pikus, Sov. Phys. JETP 73 (1990) 550.
[12] I.A. Merkulov, V.I. Perel, M.E. Portnoi, Sov. Phys. JETP 72 (1991) 669.

[13] M.I. Dyakonov, V.Yu. Kachorovskii, Sov. Phys. Semicond. 20 (1985) 110.

[14] S.D. Ganichev, S.N. Danilov, V.V. Bel'kov, E.L. Ivchenko, H. Ketterl, L.E. Vorobjev, M. Bichler, W. Wegscheider, W. Prettl, Physica E 10 (2001) 52.

[15] S.D. Ganichev, S.N. Danilov, V.V. Bel'kov, E.L. Ivchenko, M. Bichler, W. Wegscheider, D. Weiss, W. Prettl, Phys. Rev. Lett. 88 (2002) 057401. 